\title{
Analysis of metabolic pathways and fluxes in a newly discovered thermophilic and ethanol-tolerant Geobacillus strain
}

Yinjie J. Tang ${ }^{1,8}$, Rajat Sapra ${ }^{2,4}$, Dominique Joyner ${ }^{1,3}$, Terry C. Hazen ${ }^{1,3}$, Samuel Myers ${ }^{5}$, David Reichmuth $^{4}$, Harvey Blanch ${ }^{2,5,7}$, and Jay D. Keasling ${ }^{1,2,5,6,7}$

Running title: flux analysis of an ethanol tolerant thermophile

(1) Virtual Institute for Microbial Stress and Survival

(2) Joint Bio-Energy Institute, Emeryville, CA 94608

(3) Ecology Department, Lawrence Berkeley National Lab, Berkeley, 94720,

(4) Sandia National Laboratories, PO Box 969, Livermore, CA 94551-9951,

(5) Department of Chemical Engineering, University of California, Berkeley, CA 94720

(6) Department of Bioengineering, University of California, Berkeley, CA 94720

(7) Physical Biosciences Division, Lawrence Berkeley National Laboratory, Berkeley, CA 94720

(8) Department of Energy, Environmental and Chemical Engineering, Washington University, St Louis, MO63130 


\section{Abstract}

A recently discovered thermophilic bacterium, Geobacillus thermoglucosidasius

3 M10EXG, ferments a range of C5 (e.g., xylose) and C6 sugars (e.g., glucose) and is tolerant to

4 high ethanol concentrations $(10 \% \mathrm{v} / \mathrm{v})$. We have investigated the central metabolism of this

5 bacterium using both in vitro enzyme assays and ${ }^{13} \mathrm{C}$-based flux analysis to provide insights into

6 the physiological properties of this extremophile and explore its metabolism for bioethanol or

7 other bioprocess applications. Our findings show that glucose metabolism in $G$.

8 thermoglucosidasius M10EXG proceeds via glycolysis, the pentose phosphate pathway, and the

9 TCA cycle; the Entner-Doudoroff pathway and transhydrogenase activity were not detected.

10 Anaplerotic reactions (including the glyoxylate shunt, pyruvate carboxylase and

11 phosphoenolpyruvate carboxykinase) were active, but fluxes through those pathways could not

12 be accurately determined using amino acid labelling. When growth conditions were switched

13 from aerobic to micro-aerobic conditions, fluxes (based on a normalized glucose uptake rate of

14100 units $(\mathrm{gm} \mathrm{DCW})^{-1} \cdot \mathrm{hr}^{-1}$ ) through the TCA cycle and oxidative pentose phosphate pathway

15 were reduced from $64 \pm 3$ to $25 \pm 2$ and from $30 \pm 2$ to $19 \pm 2$, respectively. The carbon flux under

16 microaerobic growth was directed to ethanol, L-lactate ( $>99 \%$ optical purity), acetate, and

17 formate. Under fully anaerobic conditions, G. thermoglucosidasius M10EXG used a mixed acid

18 fermentation process and exhibited a maximum ethanol yield of $0.38 \pm 0.07 \mathrm{~mol} \mathrm{~mol}^{-1}$ glucose. In

19 silico flux balance modelling demonstrates that lactate and acetate production from $G$.

20 thermoglucosidasius M10EXG reduces the maximum ethanol yield by approximately three

21 folds, thus indicating that both pathways should be modified to maximize ethanol production.

22 Key words: C5 sugar, micro-aerobic, TCA cycle, anaplerotic pathway, flux balance model 


\section{Introduction}

A recently discovered thermophilic ethanologen, Geobacillus thermoglucosidasius

3 M10EXG (M10EXG), is a facultative anaerobe that has an optimal growth temperature of $60^{\circ} \mathrm{C}$

4 (Fong et al. 2006). It can ferment a range of C5 and C6 sugars and tolerate ethanol

5 concentrations of up to $10 \%(\mathrm{v} / \mathrm{v})$ (Fong et al. 2006), which makes it an ideal microbe for

6 improved bio-ethanol production. Moreover, Geobacillus species have many other potential

7 industrial applications for production of various thermostable enzymes, exopolysaccharides and

8 bacteriocins; they have also been found to metabolize hydrocarbons in high temperature oil

9 fields (Nazina et al. 2005) as well as degrading herbicides (such as organophosphonates)

10 (McMullan et al. 2004). However, the genome sequence of this newly discovered Geobacillus

11 strain is not yet available, and as such there is no functional genomics data. In order to engineer

12 the metabolic pathways of the bacterium for optimizing ethanol production from $\mathrm{C} 5$ and $\mathrm{C} 6$

13 sugars, an understanding of the carbon fluxes and the maximum potential for ethanol production

14 is required. In this study, we used in vitro enzyme activity assays and a ${ }^{13} \mathrm{C}$-based isotopomer

15 flux model to investigate central metabolic pathways of this thermophilic organism as a function

16 of oxygen availability (Stephanopoulos et al. 1998; Tang et al. 2007a; Tang et al. 2007b;

17 Wiechert et al. 2001). To accomplish this, cells were grown in minimal medium containing

18 either $\left[1-{ }^{13} \mathrm{C}\right]$ or $\left[2-{ }^{13} \mathrm{C}\right]$-labelled glucose as the sole carbon source, and the ${ }^{13} \mathrm{C}$-labeling patterns

19 of derivatized, intracellular amino acids were determined using gas chromatography-mass

20 spectrometry (GC-MS). An isotopomer model was then constructed to simulate all of the atom

21 transitions in the assumed biochemical network (based on both enzyme activity assay and

22 metabolic pathways of its closest sequenced species G. kaustophilus (Takami et al. 2004)) and

23 the label distribution in all central metabolites. We then searched for a set of active intracellular 
1 metabolic pathways and flux distributions that predicted the inferred isotopomer distribution of

2 key metabolites resulting from the isotopomer pattern of the derivatized amino acids. We show

3 that in the absence of genome information our approach provides an effective way to map the

4 central pathways of a new fermentative organism (Tang et al. 2007e) and directly observe the 5 functional output (i.e., metabolic fluxes) of the transcriptome, proteome, and metabolic changes 6 under different growth conditions (Sauer 2004).

\section{$8 \quad$ Materials and Methods}

Culture conditions. M10EXG was obtained from the Bacillus Genetic Stock Center at

10 the Ohio State University (Cat \# W9A44). A complete minimal medium was used (Fong et al.

11 2006) for the cell culture under defined conditions. Since singly labeled carbon substrate can

12 well resolve the Entner-Doudoroff pathway and pentose phosphate pathway, $\left[1-{ }^{13} \mathrm{C}\right] \mathrm{D}$-glucose

$13\left(10 \mathrm{~g} \mathrm{~L}^{-1}\right.$; >98\%; Cambridge Isotope, Andover, MA) was used as the sole carbon source (Fischer

14 et al. 2004). For anaerobic or micro-aerobic experiments, the cultures were incubated in sealed

15 glass bottles with septum caps, and the headspace was filled with argon (anaerobic conditions) or

16 air (micro-aerobic conditions, air liquid volume ratio 1:1). For aerobic cell cultures, cells were

17 incubated in shake flasks at $200 \mathrm{rpm}$. All cultures with labeled medium were started with a 3\%

18 inoculation volume from cells that had been first grown in Tryptic Soy Broth (BD Biosciences,

19 San Jose, CA) to stationary phase and then sub-cultured into minimal medium with 3\%

20 inoculation volume to remove the effect of naturally labelled carbon sources from the initial

21 inocula. All cultures (aerobic, microaerobic, and anaerobic) were incubated at $60^{\circ} \mathrm{C}$ with

22 shaking at $200 \mathrm{rpm}$. Total biomass growth was monitored by measuring the optical density at a

23 wavelength of $600 \mathrm{~nm}\left(\mathrm{OD}_{600}\right)$. 
Enzyme Assays. Exponentially growing cells were centrifuged and the resulting cell

2 pellets were resuspended in $1 \mathrm{ml} 100 \mathrm{mM}$ Tris- $\mathrm{HCl} \mathrm{pH} 7.4$ and lysed by sonication for all

3 enzyme assays. Total protein concentration for cell lysates was determined using the Bradford

4 method (Bio-Rad, Hercules, CA) with bovine serum albumin as the standard. All chemicals and

5 coupling enzymes were purchased from Sigma Chemical (St. Louis, MO). All enzyme assays

6 were performed at $55^{\circ} \mathrm{C}$ and monitored spectroscopically at their respective wavelengths. All

$7 \quad$ enzyme assays were performed as previously reported (McKinlay et al. 2007; Sauer et al. 2004;

8 Terada et al. 1991; Van der Werf et al. 1997). In brief, an isocitrate lyase assay contained $25 \mathrm{mM}$

9 imidazole $\mathrm{pH} 6.8,5 \mathrm{mM} \mathrm{MgCl}_{2}, 1 \mathrm{mM}$ EDTA, $4 \mathrm{mM}$ phenylhydrazine, and $1 \mathrm{mM}$ D-L-

10 isocitrate; the absorbance at $324 \mathrm{~nm}$ was used to monitor forming osazone derivatives. The

11 oxaloacetate decarboxylase assay contained $41 \mathrm{mM}$ triethanolamine-HCl (TEA) pH 8.0, $460 \mu \mathrm{M}$

$12 \mathrm{MnCl}_{2}, 300 \mu \mathrm{M} \beta-\mathrm{NADH}, 11$ units $\mathrm{mL}^{-1}$ of lactate dehydrogenase, and $2.3 \mathrm{mM}$ oxaloacetate; the

13 absorbance at $340 \mathrm{~nm}$ was used to monitor $\beta$-NADH oxidation. The $\alpha$-ketoglutarate

14 dehydrogenase assay contained $50 \mathrm{mM}$ MOPS pH 7.4, $4 \mathrm{mM} \mathrm{MgCl}, 200 \mu \mathrm{M} \mathrm{CaCl}_{2}, 6 \mathrm{mM}$

15 thiamine pyrophosphate, $6.7 \mathrm{mM} \beta-\mathrm{NAD}^{+}, 5.2 \mathrm{mM}$ cysteine- $\mathrm{HCl}$, and $25 \mathrm{mM} \alpha$-ketoglutarate;

16 the absorbance at $340 \mathrm{~nm}$ was used to monitor the increase in $\beta$-NADH. The

17 phosphoenolpyruvate (PEP) carboxylase assay contained $100 \mathrm{mM}$ Tris-acetate $\mathrm{pH} 8.5,2 \mathrm{mM}$

18 potassium $\mathrm{PEP}, 10 \mathrm{mM} \mathrm{KHCO}_{3}, 10 \mathrm{mM}$ magnesium acetate, and $1.17 \mathrm{M}$ dioxane; the

19 absorbance at $340 \mathrm{~nm}$ was used to monitor $\beta$-NADH oxidation. The transhydrogenase assay

20 contained $50 \mathrm{mM}$ Tris-HCl, $\mathrm{pH} 7.6,2 \mathrm{mM} \mathrm{MgCl}_{2}, 500 \mu \mathrm{M} \beta-\mathrm{NADH}$ and $1 \mathrm{mM}$ 3-acetylpyridine

21 adenine dinucleotide $\left(\mathrm{APAD}^{+}\right)$; the absorbance at $375 \mathrm{~nm}$ was used to monitor the loss of

$22 \mathrm{APAD}^{+}$. The PEP carboxykinase assay contained $25 \mathrm{mM}$ HEPES, pH 7.1, $50 \mathrm{mM} \mathrm{KCl,} 2 \mathrm{mM}$

$23 \mathrm{MgCl}_{2}, 50 \mathrm{mM} \mathrm{NaHCO} 3,500 \mu \mathrm{M}$ dithiothreitol, $20 \mu \mathrm{M} \beta-\mathrm{NADH}, 100 \mu \mathrm{M}$ ADP-Mg, $5 \mathrm{mM}$ 
1 glucose, 4 units $\mathrm{mL}^{-1}$ of malate dehydrogenase, 4 units $\mathrm{mL}^{-1}$ of hexokinase and $1 \mathrm{mM}$ PEP; the

2 absorbance at $340 \mathrm{~nm}$ was used to monitor $\beta$-NADH oxidation. The malic enzyme assay

3 contained $67 \mathrm{mM}$ TEA $\mathrm{pH} 7.4,3.5 \mathrm{mM}$ malic acid, $333 \mu \mathrm{M} \mathrm{NAD}(\mathrm{P})^{+}$, and $5 \mathrm{mM} \mathrm{MnCl}_{2}$; the

4 absorbance at $340 \mathrm{~nm}$ was used to monitor $\mathrm{NAD}(\mathrm{P}) \mathrm{H}$ oxidation. The pyruvate carboxylase assay

5 contained $95 \mathrm{mM}$ TEA $\mathrm{pH} 8.0,6.3 \mathrm{mM}$ pyruvate, $0.11 \%$ BSA, 26 units $\mathrm{mL}^{-1}$ malate

6 dehydrogenase, $50 \mu \mathrm{M}$ acetyl $\mathrm{CoA}, 240 \mu \mathrm{M} \beta-\mathrm{NADH}, 15 \mathrm{mM} \mathrm{KHCO}$, and $1 \mathrm{mM} \mathrm{ATP}$; the

7 absorbance at $340 \mathrm{~nm}$ was used to monitor $\beta$-NADH oxidation. All activity calculations had the

8 basal reaction rate subtracts and were normalized for amount of protein added to the assay (Table

9 1).

Analytical methods for metabolite concentrations, biomass composition, and

11 isotopomer labelling. The concentrations of glucose, formate, lactate, acetate, succinate, and

12 ethanol in the medium were measured using enzyme linked assay kits (r-Biopharm, Darmstadt,

13 Germany). Biomass constituents (proteinogenic amino acid composition) were measured by the

14 Molecular Structure Center, University of California, Davis; the fatty acids of M10EXG were

15 measured by Microbial ID (Newark, DE) (Supplementary Table S1). Most fatty acids were

16 saturated in M10EXG (a fact that would lead to decreased membrane fluidity and allow cell

17 growth at high temperatures and high ethanol concentrations (Daron 1970; Sullivan et al. 1979)),

18 and the 16- and 17-carbon fatty acids (including branched-chain iso- and anteiso-) accounted for

$19 \sim 80 \%$ of total fatty acids. The weight fractions of the various macromolecules were assumed to

20 be same as a typical bacterium: protein (52\%), RNA (16\%), DNA (3\%), lipids (9\%), and total

21 carbohydrate (17\%) (Stephanopoulos et al. 1998). The biomass constitute information was used

22 to give the estimation of range for searching the optimal fluxes to biomass pools. 
The GC-MS protocol for isotopomer measurement has been reported previously (Tang et

2 al. 2007d). In brief, protein in cell pellets (from $50 \mathrm{ml}$ culture) was hydrolyzed in $6 \mathrm{M} \mathrm{HCl}$ at

$3100^{\circ} \mathrm{C}$ for 24 hours. The resulting amino acid mixture was derivatized in $100 \mu \mathrm{L}$ tetrahydrofuran

4 (THF) and $100 \mu \mathrm{l} \mathrm{N-(tert-butyldimethylsilyl)-N-methyl-trifluoroacetamide} \mathrm{(Sigma-Aldrich,} \mathrm{St.}$

5 Louis, MO) at $70^{\circ} \mathrm{C}$ for 1 hour and analyzed using a gas chromatograph (Model 6890, Agilent,

6 Wilmington, DE) equipped with a DB5 column and a mass spectrometer (Model 5973 Network,

7 Agilent, Wilmington, Delaware). Two types of positively charged species were used in the

8 model simulation: unfragmented amino acids, $[\mathrm{M}-57]^{+}$, and fragmented amino acids that have

9 lost their $\alpha$ carboxyl group, $[\mathrm{M}-159]^{+}$. The natural abundance of isotopes was corrected using a

10 published algorithm before using the data for calculating the label distribution (Hellerstein and

11 Neese 1999).

Assumptions employed in isotopomer modelling. The development of the isotopomer

13 model was based on six assumptions. (i) A quasi-steady state is assumed to be achieved using

14 batch culture as a convenient and less expensive approach (Sauer et al. 1999; Tang et al. 2007b).

15 This assumption is based on the fact that isotopic patterns of 14 proteinogenic amino acids did

16 not change (less than $1 \%$ difference) during the exponential growth phase $\left(\mathrm{OD}_{600} 0.4-0.9\right)$. (ii)

17 The central metabolic network in M10EXG was inferred from the pathways in closely-related

18 Geobacillus kaustophilus (only sequenced Geobacillus species) and Bacillus subtilis

19 (Christiansen et al. 2002; Sauer et al. 1997). (iii) The direction of flux was based on reaction

20 thermodynamics, as suggested by a previous flux study on Bacillus subtilis (Sauer et al. 1997);

21 considerations of potential reversibility of each reaction would make the model system highly

22 underdetermined (Zhao and Shimizu 2003), thus only reactions between PEP and oxaloacetate

23 were assumed reversible. (iv) Amino acids provide isotopomer information unique to their 
1 precursors in the central metabolic pathways. To avoid possible inaccuracies resulting from

2 alternative amino acid biosynthesis routes, seven amino acids were used to determine ${ }^{13} \mathrm{C}$ fluxes

3 in central metabolism (Supplementary Table S2). These corresponding metabolites and their

4 amino acid precursors were pyruvate (alanine), acetyl-CoA (leucine), oxaloacetate (aspartic

5 acid), 2-oxo-glutarate (glutamate), phosphoenolpyruvate \& erythrose-4-phosphate

6 (phenylalanine), and 3-phosphoglycerate (serine and glycine) (Sauer et al. 1997). (v) The

7 pathways included in the model were the tricarboxylic acid (TCA) cycle, pentose phosphate (PP)

8 pathway, the Entner-Doudoroff pathway, and anaplerotic reactions. Oxalacetate decarboxylase

9 and malic enzyme were not included in the model because of no activity was observed from in

10 vitro enzyme assays (Table 1). (vi) The fluxes PEP $\leftrightarrow$ OAA and $\mathrm{Pyr} \leftarrow \rightarrow \mathrm{OAA}$ could not be

11 clearly distinguished via isotopomer labelling; therefore, PEP and pyruvate were treated as a

12 single metabolite pool and the reactions between PEP/Pyr pool and OAA were assumed

13 reversible.

14 Algorithm for ${ }^{13} \mathbf{C}$ based flux calculation. The extra-cellular fluxes (production of

15 formate, lactate, acetate and ethanol) were measured directly, and fluxes to biomass pools were

16 calculated based on the biomass composition (Supplementary Table S1). These fluxes were used

17 as inputs to the isotopomer model and tightly constrained within measurement noise. The

18 remaining unknown fluxes were determined from isotopomer fractions, to identify the operative

19 intracellular metabolic reactions as described before (Tang et al. 2007b). In brief, the complete

20 fluxes were solved using the reaction stoichiometry and atom / isotopomer mapping matrices in

21 an iterative scheme to obtain the steady-state isotopomer distributions in the intracellular

22 metabolites pools. To avoid getting trapped in a local minimum, the model applied a grid search

23 strategy (Antoniewicz et al. 2006): with the glucose uptake rate under three oxygen conditions 
1 normalized to a value of 100 units $(\mathrm{gm} \mathrm{DCW})^{-1} \mathrm{hr}^{-1}$, the model exhaustively searched all

2 combinations of independent variables (metabolite fluxes). Since the pyruvate shunt reaction

3 (pyruvate $+\mathrm{CO}_{2} \rightarrow$ oxaloacetate via pyruvate carboxylase) consumes $\mathrm{CO}_{2}$ from the medium, the

4 fraction of ${ }^{13} \mathrm{CO}_{2}$ in the medium was also estimated. The step size of the grid search algorithm

5 was 1 (normalized to the glucose uptake rate of 100 units $\left.(\mathrm{gm} \mathrm{DCW})^{-1} \mathrm{hr}^{-1}\right)$ for unknown fluxes

6 and 0.01 for the ${ }^{13} \mathrm{CO}_{2}$ fraction. All possible flux combinations were searched to determine the

7 global minima of the objective function (Tang et al. 2007b):

$$
\varepsilon\left(v_{n}\right)=\sum_{i=1}^{a}\left(\frac{M_{i}-N_{i}\left(v_{n}\right)}{\delta_{i}}\right)^{2}
$$

9 where $v_{n}$ are the unknown fluxes to be optimized in the program, $M_{i}$ are the measured MS data,

$10 N_{i}$ are the corresponding model-simulated MS data, and $\delta_{\mathrm{i}}$ are the corresponding standard

11 deviations in the GC-MS data (1 2\%). The unknown metabolic fluxes were calculated to

12 minimize $\varepsilon$. To estimate the confidence interval for calculated fluxes, a Monte Carlo approach

13 was employed (Zhao and Shimizu 2003). In brief, 20 isotopomer concentration data sets were

14 generated by addition of normally distributed measurement noise to actual measurement data.

15 The same optimization routine was used to estimate the best-fit flux distribution from these data

16 sets. Confidence limits for each flux value were obtained from the probability distribution of

17 calculated flux resulting from the simulated data sets. The model program was developed using

18 MATLAB 7.0 (The Mathworks, Natick, MA). The calculations were carried out using a Quad-

19 Process Server (Finetec, San Jose, CA) at the Lawrence Berkeley National Laboratory.

20 In silico flux balance analysis (FBA). FBA was used to estimate ethanol production

21 potential by M10EXG and prioritize the pathways for genetic engineering. Since the genome

22 sequence and functional metabolic pathway information of M10EXG were not available, in

23 silico modelling was constructed using the Simpheny platform (Genomatica, San Diego, CA) to 
1 coarsely predict the M10EXG metabolic network (Mahadevan et al. 2006), with the following

2 modifications: 1) two unique reactions found in Geobacillus strains (L-lactate dehydrogenase

3 and pyruvate carboxylase) were added to the model; 2) The biomass composition for the

4 M10EXG model is given in Supplementary Table S1. The model included $\sim 1075$ reactions and

$5 \sim 760$ constraints, and the flux calculation algorithm relied on implementing a series of

6 physicochemical constraints, including thermodynamic directionality, enzymatic capacity

7 constraints, and reaction stoichiometry constraints (Edwards and Palsson 2000a). Since the

8 number of reactions is much greater than the number of metabolites, the system requires the

9 assumption of an objective function for the in silico flux balance analysis, i.e, maximizing cell

10 growth or ethanol production (Stephanopoulos et al. 1998).

\section{Results and Discussion}

\section{Growth kinetics and cellular metabolites under various oxygen conditions. Since}

13 Geobacillus thermoglucosidasius M10EXG grows in a minimal glucose medium between 55-

$1465^{\circ} \mathrm{C}$ (its optimal growth temperature is $60^{\circ} \mathrm{C}$ ) with glucose as the sole carbon source, the

15 bacterium contains complete biosynthesis pathways for all amino acids and other essential

16 metabolites. The average doubling time in the exponential phase in minimal glucose medium

17 was two hours under aerobic growth conditions and 3.5 hours under micro-aerobic conditions

18 (Figure 1), i.e., the doubling time at high temperature was not faster than that of mesophilic

19 bacteria (such as E. coli) under similar batch conditions (Shaikh et al. 2008). Under aerobic

20 conditions, M10EXG produced $\sim 0.64 \mathrm{~mol}_{\text {acetate }}$ mol glucose ${ }^{-1}$ (Table 2). Acetate accumulation

21 in the medium can inhibit cell growth, especially for mesophilic bacteria (Lynd 1989), due to

22 change in the intracellular $\mathrm{pH}$ or inhibition of activities of key enzymes in central metabolism

23 (Luli and Strohl 1990; Tang et al. 2007c). The production of acetate by aerobically growing 
1 bacteria is often observed when the carbon source is in excess, so bacteria can regulate acetyl-

2 CoA consumption rate and quickly generate ATP when the activity of key TCA cycle enzymes

3 (e.g., citrate synthase) are inhibited (Majewski and Domach 1990). Under microaerobic or

4 anaerobic conditions, the cells secreted lactate, ethanol, and formate, in addition to acetate (Table

5 2). Under completely anaerobic conditions, the L-lactate ( $>99 \%$ optical purity) production

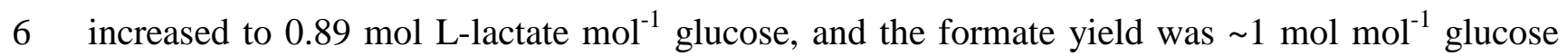

7 (i.e., pyruvate-formate lyase replaced pyruvate dehydrogenase for acetyl-CoA production), while

8 the molar yield of ethanol was $\sim 0.38 \mathrm{~mol} \mathrm{~mol}^{-1}$ glucose. The large amounts of acids produced

9 reduced the $\mathrm{pH}$ in the medium from 7.6 to $\sim$, and the cells entered the death phase (lysed) under

10 micro-aerobic condition after $20 \mathrm{hrs}$ (i.e., the $\mathrm{OD}_{600}$ dropped after $20 \mathrm{hrs}$ ). Finally, M10EXG can

11 also grow in xylose minimal medium and exhibits similar growth kinetics under different oxygen

12 conditions (Supplementary Table S3), which makes this microorganism an ideal candidate for

13 bio-ethanol production from lignocellulosic biomass (contain up to 40\% C5 sugars). However,

14 M10EXG mainly utilized glucose as carbon source when both glucose (C6) and xylose (C5)

15 were available (ratio 1:1) (Supplementary Table S4). This result indicates that the presence of

16 glucose may strongly inhibit xylose metabolism.

${ }^{13} \mathrm{C}$-based flux analysis of intra-cellular pathways under aerobic and micro-aerobic

18 conditions. Isotopomer flux models were developed based on assumed central metabolic

19 pathways to optimally fit all isotopomer data. The optimal flux distributions (based on a

20 normalized glucose uptake rate of 100 units $\left(\mathrm{gm} \mathrm{DCW}^{-1} \mathrm{hr}^{-1}\right)$ and the confidence intervals of

21 seven key intracellular fluxes (including glycolysis, PP pathway, TCA cycle and anapleurotic

22 pathways) under both aerobic and micro-aerobic conditions are shown in Figure 2. The flux

23 distribution results indicate that oxygen concentrations strongly affected the metabolic fluxes 
1 through the central pathways. Under aerobic conditions, approximately two-thirds of the glucose

2 flowed through glycolysis (relative flux $=69$ ) and the remainder through the pentose phosphate

3 pathway (flux $=30$ ), while the flux through citrate synthase (into the TCA cycle) was 64 . Under

4 micro-aerobic conditions, growth was slower $\left(0.20 \mathrm{hr}^{-1}\right)$ (Table 2), and the fluxes through TCA

5 cycle and PP pathway (G6P $\rightarrow 6 \mathrm{PG}$ ) were reduced to 25 and 19 , respectively. In vitro assays

6 showed no evidence for transhydrogenase activity under our experimental conditions (Table 1).

7 Flux through the PP pathway was sensitive to growth rate, most likely because NADPH to

8 support biomass synthesis is mainly from PP pathway (Christiansen et al. 2002). On the other

9 hand, B. subtilis showed much higher PP pathway flux $(\sim 70)$ that varied less with specific

10 growth rate (Sauer et al. 1997). This is because B. subtilis can convert excess NADPH from the

11 PP pathway to NADH via the transhydrogenase reaction and as such has greater flexibility in

12 balancing redox (Dauner et al. 2001; Sauer et al. 1997).

13 The flux results indicate that there is no Entner-Doudneroff (ED) pathway activity under

14 either experimental condition, which is consistent with the lack of a phosphogluconate

15 dehydratase (a key ED pathway enzyme) in the annotated Geobacillus kaustophilus genome

16 (Alm et al. 2005) and with the fact that Bacillus species do not use the ED pathway (Goldman

17 and Blumenthal 1963). Several anapleurotic reactions in M10EXG (as inferred from the

18 Geobacillus kaustophilus genome annotation) were present based on in vitro enzyme assays

19 (Table 1): Pyruvate $\rightarrow$ OAA (pyruvate shunt via pyruvate carboxylase), OAA $\rightarrow$ PEP (via PEP

20 carboxykinase), and PEP $\rightarrow$ OAA (via PEP carboxylase). The flux results indicate that these

21 anapleurotic reactions were down-regulated under micro-aerobic conditions: the OAA $\rightarrow$ PEP /

22 Pyruvate flux declined from 54 to 37 , and the combined flux from PEP and pyruvate to OAA

23 declined from 44 to 24 . Those non-biomass-related anapleurotic reactions may provide M10EXG 
1 central metabolism with flexibility to cope with various growth conditions (Tang et al. 2007b).

2 The glyoxylate shunt, which reduces carbon flow through the oxidative branch of the TCA cycle

3 (coupled with other anapleurotic pathways) and provides an alternative route for acetyl-CoA

4 metabolism, was also measurable under aerobic and micro-aerobic conditions in M10EXG

5 (Fischer and Sauer 2003).

6 Analysis of anaerobic pathways of M10EXG. Under anaerobic conditions, M10EXG

7 disposed part of the glucose through lactic acid and formic acid production. The formic acid

8 yield ( $\sim 1 \mathrm{~mol} \mathrm{~mole}^{-1}$ of glucose consumed) was approximately equal to the sum of the acetate

9 and ethanol yields, indicating that acetyl-CoA is a precursor to ethanol (via alcohol

10 dehydrogenase) and acetate under mixed acid fermentation (Figure 3). Based on the metabolite

11 measurements and the reported mixed acid fermentation pathway of B. subtilis (Cruz Ramos et

12 al. 2000), a simplified anaerobic pathway is proposed (Figure 3). Under anaerobic conditions,

13 carbon flux between glycolysis and the PP pathway can be directly calculated based on the

14 labelling information, because the flux ratio between the two pathways is reflected in the

15 labelling pattern of 3-phosphoglycerate (inferred from serine) and pyruvate (inferred from

16 alanine) (Sauer et al. 1997). Meanwhile, the in vitro activity of $\alpha$-ketoglutarate dehydrogenase

17 was one order magnitude lower than that measured when oxygen was available (Table 1). The

18 data showed that the enzymes of the TCA cycle were significantly repressed under anaerobic

19 conditions (Table 1) and that the TCA cycle was mainly used for biosynthesis. The major carbon

20 fluxes were directed towards mixed acids and ethanol production, which could be directly

21 measured. Eight key fluxes were shown in Figure 3: the flux was through glucose-6-phosphate

22 dehydrogenase (entrance to the oxidative branch of the PP pathway) under anaerobic conditions

23 (flux=15); pyruvate was converted to lactate via L-lactate dehydrogenase (flux=89) or to acetyl- 
1 CoA and formic acid via pyruvate formate lyase (flux=103); acetyl-CoA was mainly used for

2 ethanol (flux=38) and acetate (flux=61) production. Compared to micro-aerobic conditions,

3 formic acid production was eight-fold higher under anaerobic conditions, suggesting that

4 pyruvate formate-lyase (PFL) was induced under anaerobic conditions (note: FNR, a

5 transcriptional regulator to mediate PFL gene (Sawers and Suppmann 1992) is annotated in

6 Geobacillus kaustophilus). Under anaerobic conditions, M10EXG generated NADH primarily

7 from glycolysis (glyceraldehyde-3-phosphate dehydrogenase) (flux $=190 \pm 4$ ) and consumed

8 NADH mainly for lactate, ethanol, and acetate production (flux $=165 \pm 7$ ).

9 Verification of the isotopomer flux model. The isotopomer-based flux analysis used

10 herein is based on the labelling pattern of $\left[{ }^{13} \mathrm{C}\right]$-amino acids to infer the $\left[{ }^{13} \mathrm{C}\right]$ labelling pattern of

11 key metabolic intermediates. By tracing the path of ${ }^{13} \mathrm{C}$ from singly-labelled carbon substrate to

12 those metabolites in the pathway network, an isotopomer model can predict the carbon flux

13 distribution through central metabolism. To check the reliability of the flux analysis results, a

14 Monte Carlo method was used to calculate confidence intervals of key intracellular fluxes to

15 estimate uncertainty from measurement noise and experimental variation (as illustrated in

16 Materials and Method section). The obtained confidence intervals for seven key intracellular

17 pathways in Figure 2 showed that reaction G6P $\rightarrow 6 \mathrm{PG}$ was best determined (confidence intervals

18 for both aerobic and anaerobic conditions are within \pm 2 ), since $1-\left[{ }^{13} \mathrm{C}\right]$-glucose was good for

19 differentiating the reactions of the PP pathway from glycolysis (Fischer et al. 2004). However,

20 fluxes of anapleurotic reactions between the pyruvate/PEP pool and OAA pool had the highest

21 errors; for example, the confidence interval of OAA $\rightarrow$ PEP flux under aerobic conditions was

$22 \pm 38$. This result indicated that the isotopomer data were not sufficient to constrain these two

23 fluxes accurately. To further validate the calculated intracellular flux distribution, $\left[2{ }^{13} \mathrm{C}\right]$ 
1 glucose was used as the carbon source and the labeling of resulting key amino acids was used to

2 estimate the flux distribution under both aerobic and micro-aerobic conditions. The results from

$3\left[2-{ }^{13} \mathrm{C}\right]$ glucose experiments were qualitatively consistent with the results from $\left[1-{ }^{13} \mathrm{C}\right]$ glucose

4 experiments (Supplementary Figure S1). Fluxes through reactions of the PP pathway,

5 glycolysis, and the TCA cycle (via citrate synthase) were very similar (difference <5). In

6 contrast, the measured fluxes for PEP carboxylase, pyruvate shunt, and the glyoxylate shunt from

7 the experiments using $\left[2-{ }^{13} \mathrm{C}\right]$ glucose had larger differences (up to 10) compared to the

8 measured fluxes from experiments using $\left[1-{ }^{13} \mathrm{C}\right]$ glucose. Errors in calculated fluxes may arise

9 from several sources: 1) the isotopomer information may be insufficient to constrain certain

10 anapleurotic reactions very accurately; 2) measurement uncertainty of extracellular metabolites

11 in batch cultures; 3) protein degradation and reincorporation of metabolites from catabolized

12 amino acids into metabolic intermediates.

13 In silico analyses of metabolic network for ethanol production. Since genetic

14 engineering of thermophilic bacteria is very difficult, it will, therefore, be beneficial to know

15 which reactions are the most important targets for genetic manipulation to improve ethanol

16 production. As such, an in silico flux balance analysis (FBA) was performed to coarsely predict

17 the optimal cellular metabolism for ethanol production via Simpheny Software from Genomatica

18 (San Diego, CA) (Mahadevan et al. 2006). The FBA model did not require isotopomer

19 information or detailed kinetic parameters for individual metabolic reactions (Edwards and

20 Palsson 2000a; Edwards and Palsson 2000b). Although these models are underdetermined and

21 may not reflect the actual metabolic flux distribution if typical objective function is assumed

22 without any additional constraints (e.g., maximum biomass production, Supplementary Figure

23 S2), they have proven to be a useful tool to provide important guidelines to explore the target 
1 pathways for genetic engineering (Stephanopoulos et al. 1998). The theoretical maximum yields

2 of acetate, ethanol, lactate, formate, and biomass, as well as the theoretical maximum growth rate

3 (assuming an average carbon substrate uptake rate of $5 \mathrm{mM}$ glucose $(\mathrm{gm} \mathrm{DCW})^{-1} \mathrm{hr}^{-1}$ ), were

4 estimated using the Simpheny model. The predicted maximum yields of the metabolites and

5 biomass were much higher than the corresponding measured yields (Table 2). A plot of the

6 theoretical maximum ethanol production rate as the function of lactate and acetate production

7 rates for two growth rates $\left(0.1 \mathrm{hr}^{-1}\right.$ and $\left.0.2 \mathrm{hr}^{-1}\right)$, with or without formate production, indicates

8 that mixed acid production or a high growth rate significantly reduce the ethanol production rate

9 (Figure 4), because the mixed acid fermentation and biomass growth compete for the precursors

10 and reducing power (NADH) with ethanol production. Lactate production (by L-lactate

11 dehydrogenase) has the largest impact on ethanol yield followed by acetate production (acetate

12 kinase and phosphotransacetylase), while formate production (by pyruvate-formate lyase) has the

13 least impact on ethanol yield.

14 When growth rate maximization was used as the objective function, model results (Figure

155 , the three dotted arrows linking the measured ethanol fluxes with the corresponding measured

16 growth rates) indicated that ethanol production by M10EXG was much lower than the theoretical

17 value. Meanwhile, the growth rate and all fluxes through reactions of the PP pathway and TCA

18 cycle declined when more ethanol production was specified (Figure 5). The TCA cycle and

19 oxidative PP pathway appeared to be the most sensitive to ethanol production, indicated by the

20 slopes of the fluxes through each pathway as a function of ethanol production. Those pathways

21 must be sufficiently down-regulated in order to produce high levels of ethanol. On the other

22 hand, the non-oxidative PP pathway ( $\mathrm{G} 3 \mathrm{P}+\mathrm{S} 7 \mathrm{P} \rightarrow \mathrm{E} 4 \mathrm{P}+\mathrm{F} 6 \mathrm{P}$ via transaldolase) appeared to be

23 relatively insensitive to ethanol production. 


\section{Summary}

The rising cost and use of fossil fuels has renewed focus on lignocellulosic ethanol (Lin

3 and Tanaka 2006) production via simultaneous saccharification and fermentation process (SSF)

4 (Lin and Tanaka 2006). However, enzymes employed to hydrolyze lignocellulosic biomass to

5 simpler sugars for fermentation generally have temperature optimum of around $55^{\circ} \mathrm{C}$, whereas

6 the industrial organisms used to ferment the sugars to ethanol or other products (e.g.,

7 Saccharomyces cerevisiae (Antoni et al. 2007) and E. coli (Dien et al. 2003)) have a lower

8 operating temperature. Secondly, yeast cannot typically ferment C5 sugars (Sonderegger et al.

9 2004) such as xylose, a major component of lignocellulosic biomass. Furthermore, common

10 thermophilic ethanologens, e.g., Clostridium thermosaccharolyticum, are strict anaerobes (Lin

11 and Tanaka 2006) and cannot tolerate high ethanol concentrations (>4\% w/v) (Fong et al. 2006).

12 Geobacillus thermoglucosidasius M10EXG overcomes some of these aforementioned limitations

13 and has many potential advantages for ethanol or other bio-product production: it tolerates high

14 ethanol concentrations $(>10 \% \mathrm{v} / \mathrm{v}$ ); it can utilize a wide range of substrates (particularly

15 pentoses and insoluble substrates), which makes it an attractive organism for simultaneous

16 saccharification and fermentation of lignocellulosic biomass; there are lower risks of

17 contamination by other microorganisms (Akao et al. 2007) due to growth at high temperatures;

18 the growth medium will have desirable properties at high temperatures (reduced viscosity,

19 increased diffusion rates and substrate solubility, reduced energy requirements for mixing, and

20 the possibility of combining the fermentation and distillation processes to continuously extract

21 ethanol) (Lin and Tanaka 2006; Lynd 1989). This study investigates this species' metabolic

22 network via in vitro enzyme assays and ${ }^{13} \mathrm{C}$ based flux analysis. The obtained information 
1 provides guidelines for engineering the metabolic pathways for bioethanol production as well as

2 other environmental and industrial applications.

\section{Acknowledgements}

4 We thank Dr. Steve Van Dien (Genomatica) for helping with the Simpheny model and

5 Jeannie Chu for helping with metabolite measurement. Financial support for this research was

6 provided by the Sandia National Laboratories Laboratory Directed Research and Development

7 Program. Sandia is a multi-program laboratory operated by the Sandia Corporation, a Lockheed

8 Martin Company, for the United States Department of Energy under Contract DE-AC04-

9 94AL85000. D.J. and T.C. H. acknowledges support by the Virtual Institute for Microbial Stress

10 and Survival (http://VIMSS.lbl.gov) supported by the U.S. Department of Energy, Office of

11 Science, Office of Biological and Environmental Research, Genomics: GTL Program through

12 contract DE-AC02-05CH11231 between the Lawrence Berkeley National Laboratory and the US

13 Department of Energy. This work is also a part of the Joint BioEnergy Institute supported by the

14 U.S. Department of Energy.

\section{Financial Interest}

16 Jay D. Keasling has a consulting relationship with and a financial interest in Amyris and 17 a financial interest in LS9, two biofuel companies. 
Akao S, Tsuno H, Cheon J. 2007. Semi-continuous L-lactate fermentation of garbage without sterile condition and analysis of the microbial structure. Water Research 41:1774-1780.

Alm EJ, Huang KH, Price MN, Koche RP, Keller K, Dubchak IL, Arkin AP. 2005. The MicrobesOnline Web site for comparative genomics. Genome Res. 15:1015-1022.

Antoni D, Zverlov VV, Schwarz WH. 2007. Biofuels from microbes. Appl Microbiol Biotechnol. 77(1):23-35.

Antoniewicz MR, Kelleher JK, Stephanopoulos G. 2006. Determination of confidence intervals of metabolic fluxes estimated from stable isotope measurements. Metabolic Engineering 8(4):324-37.

Christiansen T, Christensen B, Nielsen J. 2002. Metabolic network analysis of Bacillus claussi on minimal and semirich medium using ${ }^{13} \mathrm{C}$-Labeled glucose. Metabolic Engineering(4):159-169.

Cruz Ramos H, Hoffmann T, Marino M, Nedjari H, Presecan-Siedel E, Dreesen O, Glaser P, Jahn D. 2000. Fermentative Metabolism of Bacillus subtilis: Physiology and Regulation of Gene Expression. Journal of Bacteriology 182(11):3072-80.

Daron HH. 1970. Fatty Acid Composition of Lipid Extracts of a Thermophilic Bacillus Species. Journal of Bacteriology 101(1):145-151.

Dauner M, Bailey JE, Sauer U. 2001. Metabolic flux analysis with a comprehensive isotopomer model in Bacillus subtilis. Biotechnology and Bioengineering 76(2):144-156.

Dien BS, Cotta MA, Jeffries TW. 2003. Bacteria engineered for fuel ethanol production: current status. Appl Microbiol Biotechnol. 63(3):258-66.

Edwards JS, Palsson BO. 2000a. The Escherichia coli MG1655 in silico metabolic genotype: Its definition, characteristics, and capabilities. PNAS 97(10):5528-5533.

Edwards JS, Palsson BO. 2000b. Robustness Analysis of the Escherichia coli Metabolic Network. Biotechnology Progress 16:927-939.

Fischer E, Sauer U. 2003. A novel metabolic cycle catalyzes glucose oxidation and anaplerosis in hungry Escherichia coli. J Biol Chem. 278(47):46446-51.

Fischer E, Zamboni N, Sauer U. 2004. High-throughput metabolic flux analysis based on gas chromatography-mass spectrometry derived 13C constraints. Analytical Biochemistry 325:308-316.

Fong JC, Svenson CJ, Nakasugi K, Leong CT, Bowman JP, Chen B, Glenn DR, Neilan BA, Rogers PL. 2006. Isolation and characterization of two novel ethanol-tolerant facultativeanaerobic thermophilic bacteria strains from waste compost. Extremophiles 10(5):363-72.

Goldman M, Blumenthal HJ. 1963. Pathways of glucose catabolism in Bacillus subtilis. Journal of Bacteriology 86:303-11.

Hellerstein MK, Neese RA. 1999. Mass isotopomer distribution analysis at eight years: theoretical, analytic, and experimental considerations. American Journal of PhysiologyEndocrinology and Metabolism 276(6):E1146-E1170.

Lin Y, Tanaka S. 2006. Ethanol fermentation from biomass resources: current state and prospects. Applied Microbiology and Biotechnology 69:627-642.

Luli GW, Strohl WR. 1990. Comparison of growth, acetate production, and acetate inhibition of Escherichia coli strains in batch and fed-batch fermentations. Applied and Environmental Microbiology 56(4):1004-1011. 
Lynd LR. 1989. Production of Ethanol from Lignocellulosic Materials Using Thermophilic Bacteria: Critical Evaluation of Potential and Review. Fiechter A, editor. Heidelberg: Springer-Verlag. 1-52 p.

Mahadevan R, Bond DR, Butler JE, Esteve-Nunez A, Coppi MV, Palsson BO, Schilling CH, Lovley DR. 2006. Characterization of metabolism in the Fe(III)-reducing organism Geobacter sulfurreducens by constraint-based modeling. Applied and Environmental Microbiology 72(2):1558-68.

Majewski RA, Domach MM. 1990. Simple Constrained-Optimization View of Acetate Overflow in E. coli. Biotechnology and Bioengineering 35(7):732-38.

McKinlay JB, Shachar-Hill Y, Zeikus JG, Vieille C. 2007. Determining Actinobacillus succinogenes metabolic pathways and fluxes by NMR and GC-MS analyses of ${ }^{13} \mathrm{C}$ labeled metabolic product isotopomers. Metabolic Engineering 9(2):177-192.

McMullan G, Christie JM, Rahman TJ, Banat IM, Ternan NG, Marchant R. 2004. Habitat, applications and genomics of the aerobic, thermophilic genus Geobacillus. Biochemical Society Transactions 32(2):214-7.

Nazina TN, Sokolova DSh, Grigoryan AA, Shestakova NM, Mikhailova EM, Poltaraus AB, Tourova TP, Lysenko AM, Osipov GA, Belyaev SS. 2005. Geobacillus jurassicus sp. nov., a new thermophilic bacterium isolated from a high-temperature petroleum reservoir, and the validation of the Geobacillus species. Syst. Appl. Microbiol. 28(1):43-53.

Sauer U. 2004. High-throughput phenomics: experimental methods for mapping fluxomes. Current Opinion in Biotechnology 15:58-63.

Sauer U, Canonaco F, Heri S, Perrenoud A, Fischer E. 2004. The soluble and membrane-bound transhydrogenases UdhA and PntAB have divergent functions in NADPH metabolism of Escherichia coli. Journal of Biological Chemistry 279(8):6613-6619.

Sauer U, Hatzimanikatis V, Bailey JE, Hochuli M, Szyperski T, Wuthrich K. 1997. Metabolic fluxes in riboflavin-producing Bacillus subtilis. Nature Biotechnology 15(5):448-52.

Sauer U, Lasko DR, Fiaux J, Hochuli M, Glaser R, Szyperski T, Wuthrich K, Bailey JE. 1999. Metabolic flux ratio analysis of genetic and environmental modulations of Escherichia coli central carbon metabolism. Journal of Bacteriology 181(21):6679-6688.

Sawers G, Suppmann B. 1992. Anaerobic Induction of Pyruvate Formate-Lyase Gene Expression Is Mediated by the ArcA and FNR Proteins. Journal of Bacteriology 174(11):3474-3478.

Shaikh AS, Tang YJ, Mukhopadhyay A, Keasling JD. 2008. Isotopomer Distributions in Amino Acids from a Highly Expressed Protein as a Proxy for Those from Total Protein. Anal Chem. 80 (3):886-890.

Sonderegger M, Jeppsson M, Larsson C, Gorwa-Grauslund MF, Boles E, Olsson L, SpencerMartins I, Hahn-Hägerdal B, Sauer U. 2004. Fermentation performance of engineered and evolved xylose-fermenting Saccharomyces cerevisiae strains. Biotechnol Bioeng. 87(1):90-8.

Stephanopoulos GN, Aristidou AA, Nielsen J. 1998. Metabolic Engineering Principles and Methodologies. San Diego: Academic Press. 75, 120-130 p.

Sullivan KH, Hegeman GD, Cordes EH. 1979. Alteration of the Fatty Acid Composition of Escherichia coli by Growth in the Presence of Normal Alcohols. Journal of Bacteriology 138(1):133-138. 
Takami H, Takaki Y, Chee GJ, Nishi S, Shimamura S, Suzuki H, Matsui S, Uchiyama I. 2004. Thermoadaptation trait revealed by the genome sequence of thermophilic Geobacillus kaustophilus. Nucleic Acids Res. 32(21):6292-6303.

Tang YJ, Chakraborty R, Martin HG, Chu J, Hazen TC, Keasling JD. 2007a. Flux analysis of central metabolic pathways in Geobacter metallireducens during reduction of soluble Fe(III)-NTA. Applied and Environmental Microbiology 73(12):3859-3864.

Tang YJ, Hwang JS, Wemmer D, Keasling JD. 2007b. The Shewanella oneidensis MR-1 fluxome under various oxygen conditions. Applied and Environmental Microbiology 73(3):718-29.

Tang YJ, Meadows AL, Keasling JD. 2007c. A kinetic model describing Shewanella oneidensis MR-1 growth, substrate consumption, and product secretion. Biotechnology and Bioengineering 189(3):894-901.

Tang YJ, Meadows AL, Kirby J, Keasling JD. 2007d. Anaerobic central metabolic pathways in Shewanella oneidensis MR-1 reinterpreted in the light of isotopic metabolite labeling. Journal of Bacteriology 189(3):894-901.

Tang YJ, Pingitore F, Mukhopadhyay A, Phan R, Hazen TC, Keasling JD. 2007e. Pathway confirmation and flux analysis of central metabolic pathways in Desulfovibrio vulgaris Hildenborough using GC-MS and FT-ICR mass spectrometry. Journal of Bacteriology 189(3):940-949.

Terada K, Murata T, Izui K. 1991. Site-Directed Mutagenesis of Phosphoenolpyruvate Carboxylase from E. coli: The Role of $\mathrm{His}^{579}$ in the Catalytic and Regulatory Functions. J. Biochem. 109:49 - 54.

Van der Werf MJ, Guettler MV, Jain MK, Zeikus JG. 1997. Environmental and physiological factors affecting the succinate product ratio during carbohydrate fermentation by Actinobacillus sp. 130Z. Arch Microbiol. 167(6):332-342.

Wiechert W, Mollney M, Petersen S, de Graaf AA. 2001. A Universal Framework for ${ }^{13} \mathrm{C}$ Metabolic Flux Analysis. Metabolic Engineering 3:265-283.

Zhao J, Shimizu K. 2003. Metabolic flux analysis of Escherichia coli K12 grown on 13C-labeled acetate and glucose using GC-MS and powerful flux calculation method. Journal of Biotechnology 101:101-117. 


\section{Figure and Table Captions}

2 Figure 1: Growth kinetics of M10EXG under three oxygen conditions: $\square$ aerobic, $\diamond$ micro-

3 aerobic, $\circ$ anaerobic.

4

5 Figure 2. Pathways and flux distributions of glucose metabolism under aerobic (top) and micro-

6 aerobic (bottom) conditions. The amino acids used for isotopomer models were shown in

7 parentheses. The glucose uptake rates were normalized to a value of 100 . Dotted lines indicate

8 that the pathways are not active. Abbreviations: Acetyl-CoA, acetyl-coenzyme A; CIT, citrate;

9 E4P, erythrose-4-phosphate; C1, 5,10-Me-THF; F6P, fructose-6-phosphate; G6P, glucose-6-

10 phosphate; 6PG, 6-phosphogluconate; ICT, isocitrate; MAL, malate; OAA, oxaloacetate; OXO,

11 2-oxoglutarate; PEP, phosphoenolpyruvate; PGA, 3-phosphoglycerate; C5P, ribose-5-phosphate

12 (or ribulose-5-phosphate or xylulose-5-phosphate); S7P, sedoheptulose-7-phosphate; SUC,

13 succinate; T3P, triose-3-phosphate.

14

15 Figure 3. M10EXG mixed acid fermentation. The abbreviations were the same as those in

16 Figure 2. Key reactions (and their corresponding relative fluxes): 1. glucose-6-phosphate

17 isomerase; 2. glucose-6-phosphate dehydrogenase; 3. T3P dehydrogenase; 4. L-lactate

18 dehydrogenase; 5. pyruvate-formate lyase; 6. acetaldehyde dehydrogenase; 7. alcohol

19 dehydrogenase; 8. phosphate acetyltransferase/acetate kinase. The arrows were drawn in

20 proportion to the fluxes. Fluxes below $10 \%$ of the glucose uptake rate were represented by non-

21 scaled hairlines. 
1 Figure 4. Effect of mixed acids production and biomass growth rate on ethanol production as

2 calculated by the FBA model. The glucose uptake rate was set to $5 \mathrm{mmol} \mathrm{hr}^{-1} \mathrm{~g}^{-1}$ biomass. The

3 units for ethanol and acids production rates are mmol hr${ }^{-1} \mathrm{~g}^{-1}$ biomass. (a) Growth rate $=0.1 \mathrm{hr}^{-1}$,

4 formate production $=0$. (b) Growth rate $=0.2 \mathrm{hr}^{-1}$, formate production $=0$. (c) Growth rate $=$

$50.1 \mathrm{hr}^{-1}$, formate production was assumed to equal the sum of the ethanol and acetate production

6 rates; (d) Growth rate $=0.2 \mathrm{hr}^{-1}$, formate production was assumed to equal the sum of the ethanol

7 and acetate production rates.

9 Figure 5. Change in central metabolism as a function of ethanol production as predicted by the 10 in silico flux balance model (Simpheny). The objective function used for the calculations was the 11 maximal biomass production. Symbols: growth rate (०); flux into the TCA cycle via citrate 12 synthase ( $\boldsymbol{\Delta}$ ); flux into the pentose phosphate pathway via glucose 6-phosphate dehydrogenase

$13(\bullet)$ and via transaldolase $(\mathrm{GAP}+\mathrm{S} 7 \mathrm{P} \rightarrow \mathrm{E} 4 \mathrm{P}+\mathrm{F} 6 \mathrm{P})(\bullet)$; flux through the pyruvate shunt $(\boldsymbol{\bullet})$. The

14 three dashed arrows linked the measured ethanol flux values with their corresponding measured 15 growth rates for the three growth conditions (aerobic, micro-aerobic, and anaerobic). The fact 16 that the lines (skewed dashed arrows) were not vertical indicates a difference between in silico 17 model predicted flux (optimal metabolism) and experimentally measured flux (actual 18 metabolism).

20 Table 1. Enzyme activities in cell extracts of Geobacillus thermoglucosidasius M10EXG under 21 three oxygen conditions $(n=3)$. 
1 Table 2. Growth kinetics and yields of ethanol and organic acids under the three oxygen 2 conditions: aerobic growth $\left(\mathrm{G}+\mathrm{O}_{2}\right)$, micro-aerobic growth $\left(\mathrm{G}+\mu \mathrm{O}_{2}\right)$, anaerobic growth $(\mathrm{G}$ $\left.3 \mathrm{O}_{2}\right)$. 
Figures

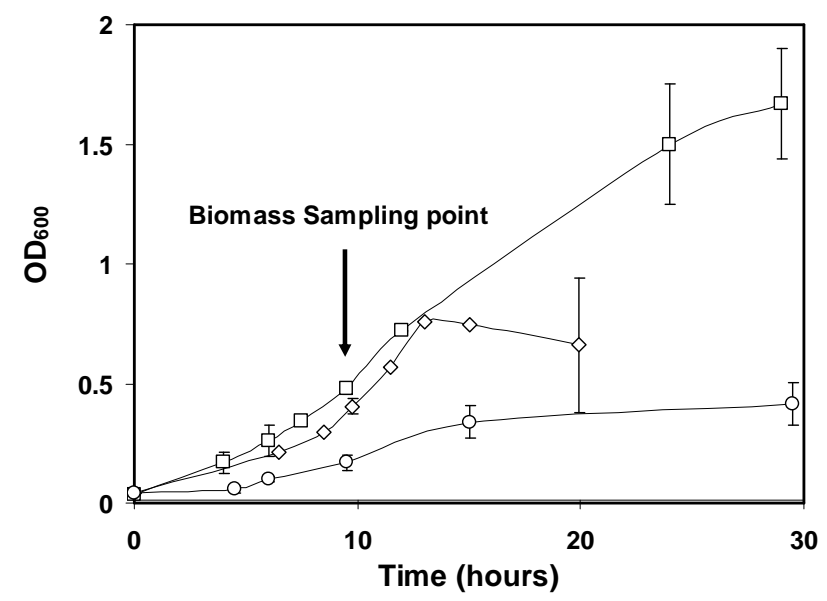

Figure 1. Growth kinetics of M10EXG under three oxygen conditions: $\square$ aerobic, $\diamond$ microaerobic, o anaerobic. 


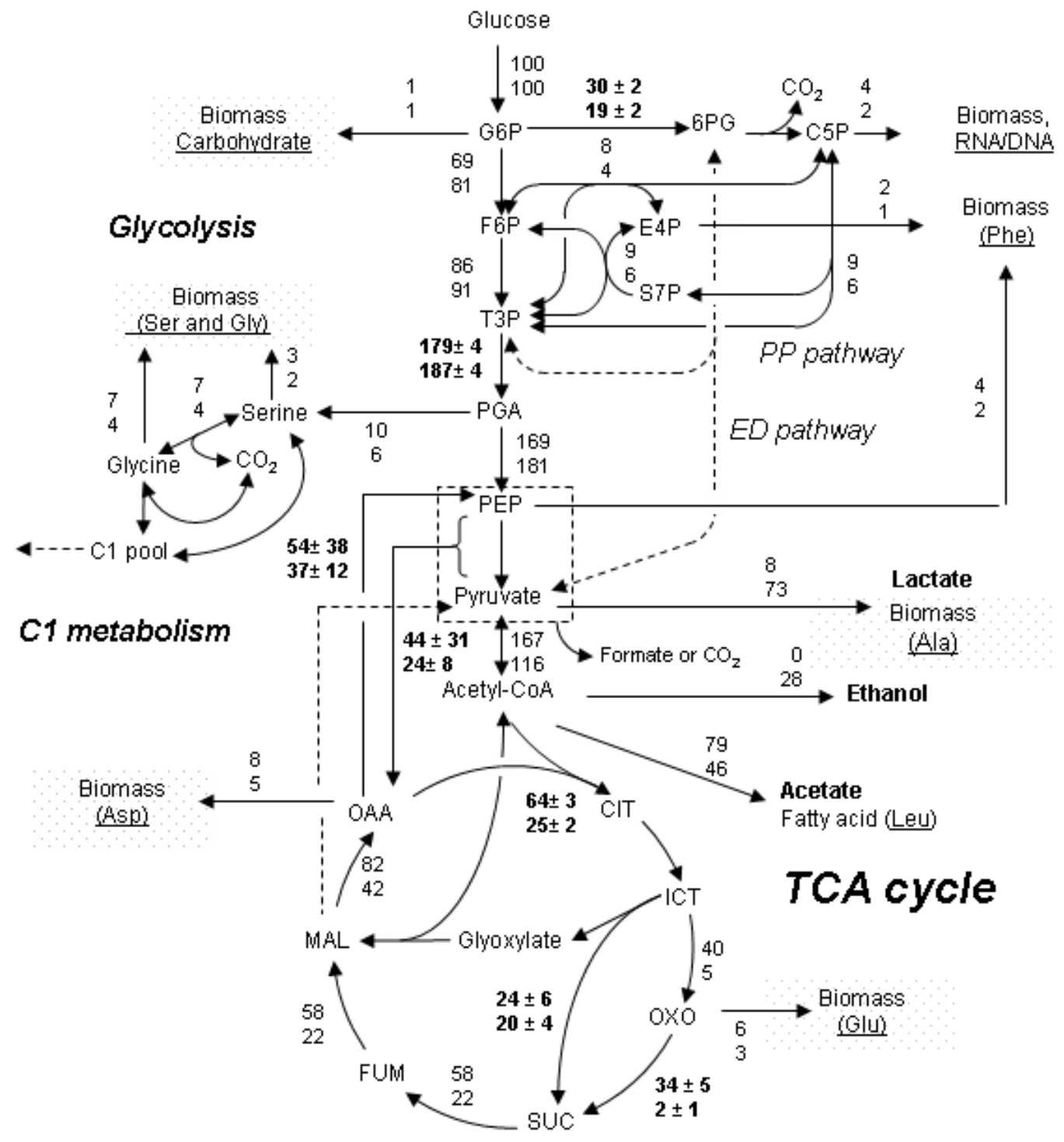

Figure 2. Pathways and flux distributions of glucose metabolism under aerobic (top) and microaerobic (bottom) conditions. The amino acids used for isotopomer models were shown in parentheses. The glucose uptake rates were normalized to a value of 100. Dotted lines indicate that the pathways are not active. Abbreviations: Acetyl-CoA, acetyl-coenzyme A; CIT, citrate; E4P, erythrose-4-phosphate; C1, 5,10-Me-THF; F6P, fructose-6-phosphate; G6P, glucose-6phosphate; 6PG, 6-phosphogluconate; ICT, isocitrate; MAL, malate; OAA, oxaloacetate; OXO, 2-oxoglutarate; PEP, phosphoenolpyruvate; PGA, 3-phosphoglycerate; C5P, ribose-5-phosphate (or ribulose-5-phosphate or xylulose-5-phosphate); S7P, sedoheptulose-7-phosphate; SUC, succinate; T3P, triose-3-phosphate. 


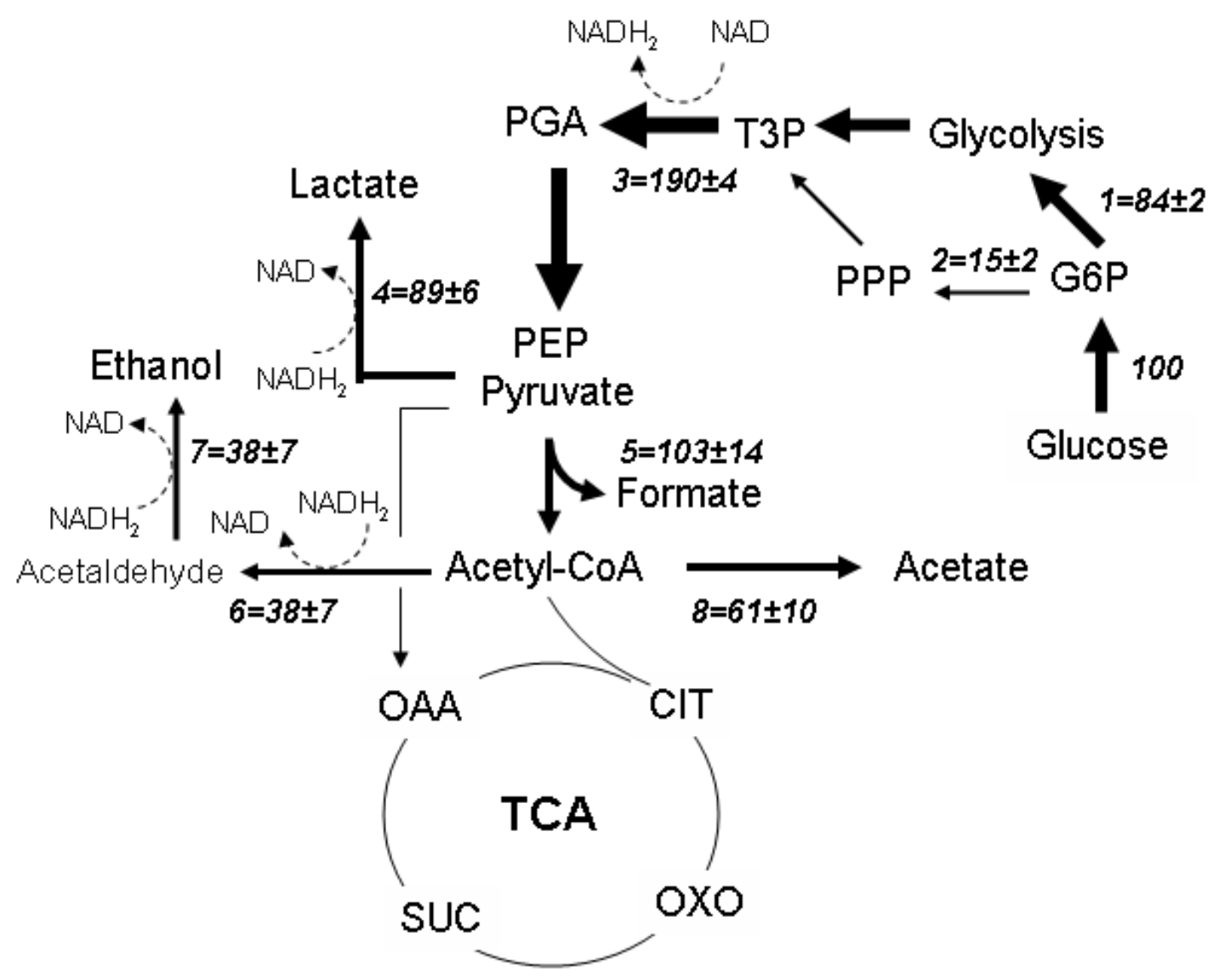

Figure 3. M10EXG mixed acid fermentation. The abbreviations were the same as those in Figure 2. Key reactions (and their corresponding relative fluxes): 1. glucose-6-phosphate isomerase; 2. glucose-6-phosphate dehydrogenase; 3. T3P dehydrogenase; 4. L-lactate dehydrogenase; 5. pyruvate-formate lyase; 6. acetaldehyde dehydrogenase; 7. alcohol dehydrogenase; 8. phosphate acetyltransferase/acetate kinase. The arrows were drawn in proportion to the fluxes. Fluxes below $10 \%$ of the glucose uptake rate were represented by nonscaled hairlines. 

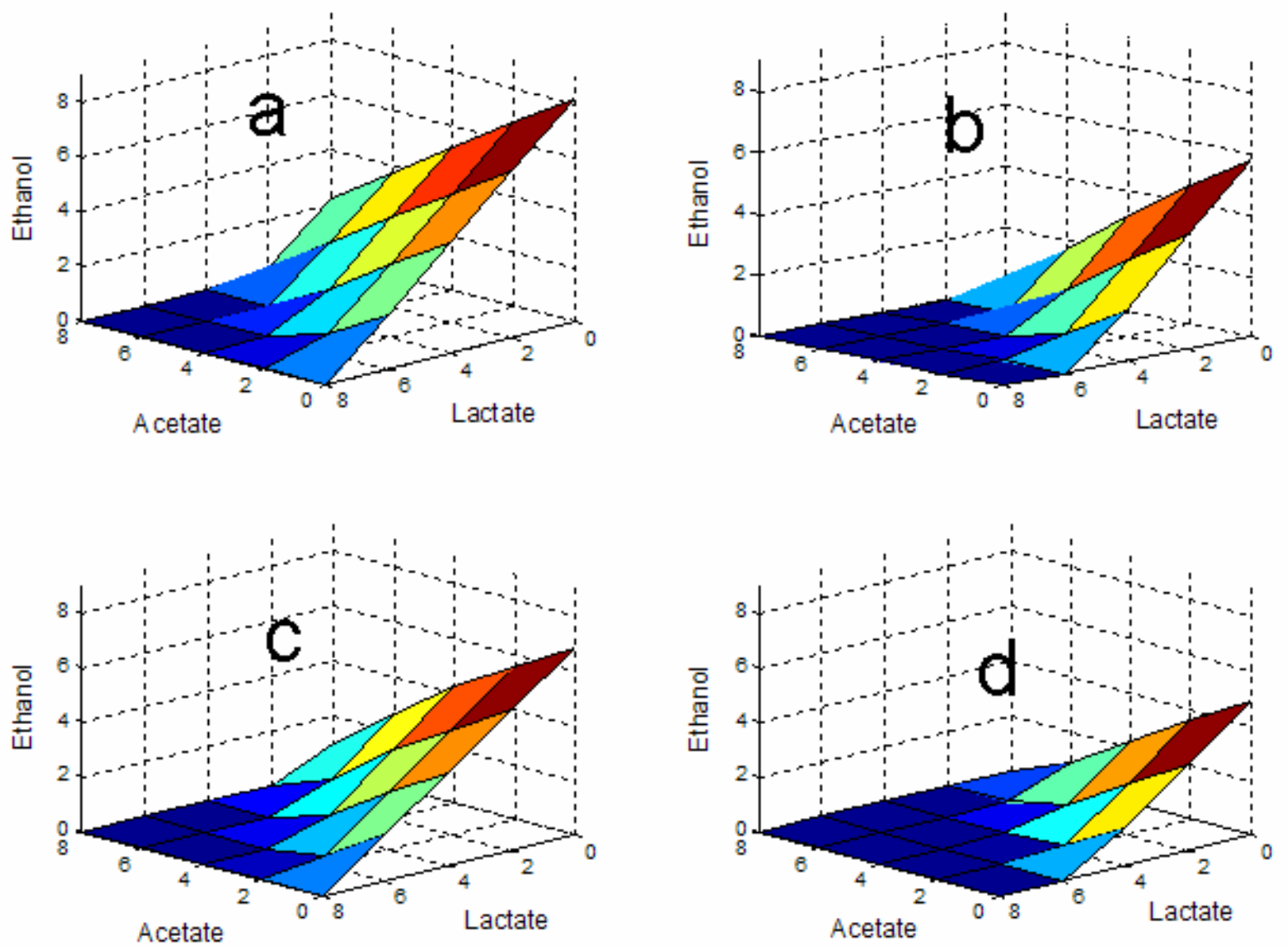

Figure 4. Effect of mixed acids production and biomass growth rate on ethanol production as calculated by the FBA model. The glucose uptake rate was set to $5 \mathrm{mmol} \mathrm{hr}^{-1} \mathrm{~g}^{-1}$ biomass. The units for ethanol and acids production rates are mmol hr${ }^{-1} \mathrm{~g}^{-1}$ biomass. (a) Growth rate $=0.1 \mathrm{hr}^{-1}$, formate production $=0$. (b) Growth rate $=0.2 \mathrm{hr}^{-1}$, formate production $=0$. (c) Growth rate $=$ $0.1 \mathrm{hr}^{-1}$, formate production was assumed to equal the sum of the ethanol and acetate production rates; (d) Growth rate $=0.2 \mathrm{hr}^{-1}$, formate production was assumed to equal the sum of the ethanol and acetate production rates. 


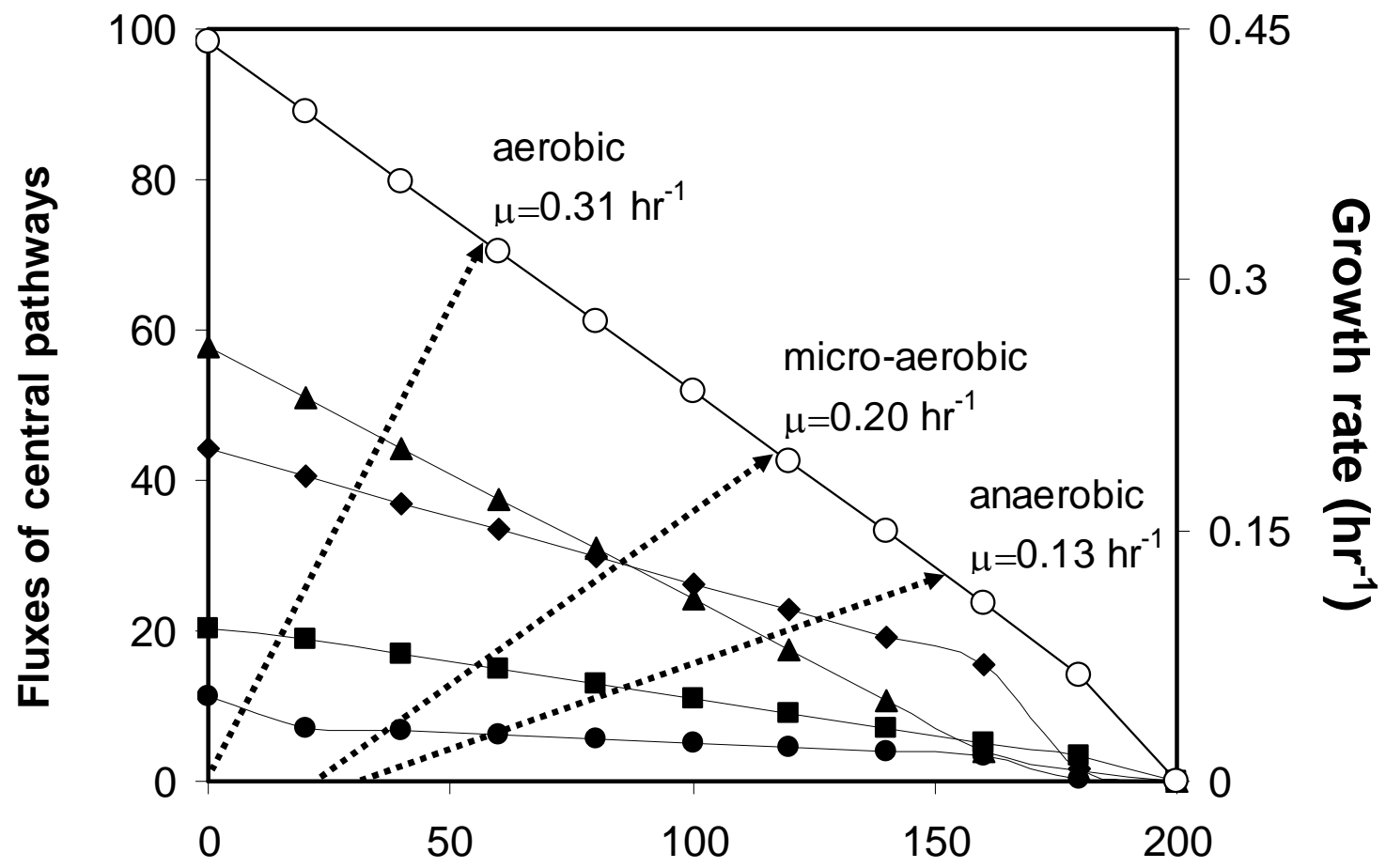

\section{Ethanol fluxes}

Figure 5. Change in central metabolism as a function of ethanol production as predicted by the in silico flux balance model (Simpheny). The objective function used for the calculations was the maximal biomass production. Symbols: growth rate (O); flux into the TCA cycle via citrate synthase $(\boldsymbol{\Delta})$; flux into the pentose phosphate pathway via glucose 6-phosphate dehydrogenase $(\diamond)$ and via transaldolase $(\mathrm{GAP}+\mathrm{S} 7 \mathrm{P} \rightarrow \mathrm{E} 4 \mathrm{P}+\mathrm{F} 6 \mathrm{P})(\bullet)$; flux through the pyruvate shunt $(\boldsymbol{\bullet})$. The three dashed arrows linked the measured ethanol flux values with their corresponding measured growth rates for the three growth conditions (aerobic, micro-aerobic, and anaerobic). The fact that the dashed arrows were not vertical indicated a difference between in silico model predicted fluxes (optimal metabolism) and experimentally measured fluxes (actual metabolism). 


\section{Tables}

Table 1. Enzyme activities in cell extracts of Geobacillus thermoglucosidasius M10EXG under three oxygen conditions $(\mathrm{n}=3)$.

\begin{tabular}{llccc}
\hline Enzymes & EC number & \multicolumn{3}{c}{ Specific activity (units g protein ${ }^{-1}$ ) } \\
& & Aerobic & Micro-aerobic & Anaerobic \\
\hline Oxalacetate decarboxylase $\left(-\mathrm{Na}^{+}\right)$ & EC 4.1.1.3 & 0 & $3 \pm 3$ & 0 \\
Oxalacetate decarboxylase $\left(+\mathrm{Na}^{+}\right)$ & EC 4.1 .1 .3 & 0 & $2 \pm 3$ & 0 \\
Malic Enzyme (NADP+) & EC 1.1.1.40 & 0 & 0 & 0 \\
Malic Enzyme (NAD+) & EC 1.1.1.38 & 0 & 0 & 0 \\
$\alpha$-ketoglutarate dehydrogenase & EC 1.2.4.2 & $230 \pm 52$ & $92 \pm 75$ & $26 \pm 18$ \\
Pyruvate carboxylase & EC 6.4.1.1 & $682 \pm 385$ & $660 \pm 279$ & $615 \pm 246$ \\
PEP carboxykinase & EC 4.1.1.49 & $249 \pm 50$ & $373 \pm 25$ & $298 \pm 22$ \\
Isocitrate lyase & EC 4.1.3.1 & $26 \pm 2$ & $22 \pm 4$ & $21 \pm 5$ \\
PEP carboxylase & EC 4.1.1.31 & $89 \pm 58$ & $79 \pm 70$ & $49 \pm 21$ \\
Transhydrogenase & EC 1.6.1.1. & 0 & 0 & 0 \\
\hline
\end{tabular}

Note: one unit catalyzes the formation of one $\mu \mathrm{mol}$ of substrate per minute. 
Table 2. Growth kinetics and yields of ethanol and organic acids under the three oxygen conditions: aerobic growth $\left(\mathrm{G}+\mathrm{O}_{2}\right)$, micro-aerobic growth $\left(\mathrm{G}+\mu \mathrm{O}_{2}\right)$, anaerobic growth $(\mathrm{G}$ $\mathrm{O}_{2}$ ).

\begin{tabular}{lllll}
\hline Yield $^{1}$ & $\mathrm{G}+\mathrm{O}_{2}$ & $\mathrm{G}+\mu \mathrm{O}_{2}$ & $\mathrm{G}^{1} \mathrm{O}_{2}{ }^{2}$ & $\mathrm{Max}^{3}$ \\
\hline $\mathrm{Y}_{\text {ace/s }}$ & $0.64 \pm 0.12$ & $0.40 \pm 0.05$ & $0.61 \pm 0.10$ & 2.6 \\
$\mathrm{Y}_{\text {lact/s }}$ & $0.02 \pm 0.01$ & $0.67 \pm 0.07$ & $0.89 \pm 0.06$ & 2 \\
$\mathrm{Y}_{\text {etho/s }}$ & $0.01 \pm 0.01$ & $0.28 \pm 0.04$ & $0.38 \pm 0.07$ & 2 \\
$\mathrm{Y}_{\text {form/s }}$ & 0 & $0.13 \pm 0.05$ & $1.03 \pm 0.14$ & 5.6 \\
$\mathrm{Y}_{\text {biomass/s }}$ & $0.27 \pm 0.05$ & $0.19 \pm 0.04$ & $0.08 \pm 0.03$ & 0.34 \\
\hline Growth rate, $\mathrm{hr}^{-1}$ & $0.31 \pm 0.04$ & $0.20 \pm 0.04$ & $0.13 \pm 0.03$ & 0.44 \\
\hline
\end{tabular}

${ }^{1}$ Metabolite yield unit, mol metabolites mol $^{-1}$ glucose. Biomass yield unit, DCW g ${ }^{-1}$ glucose.

${ }^{2} \mathrm{~A}$ small amount of succinate was also detected.

${ }^{3}$ The maximum yield for each metabolite was predicted using Simpheny. The model assumed a glucose uptake rate equal to $5 \mathrm{mM} \mathrm{hr}^{-1} \mathrm{DCW}^{-1}$. 